\title{
FACTORING WEAKLY COMPACT HOMOMORPHISMS, INTERPOLATION OF BANACH ALGEBRAS AND MULTILINEAR INTERPOLATION
}

\author{
FERNANDO COBOS \\ Departamento de Análisis Matemático, Facultad de Matemáticas \\ Universidad Complutense de Madrid \\ 28040 Madrid, Spain \\ E-mail:cobos@mat.ucm.es \\ LUZ M. FERNÁNDEZ-CABRERA \\ Sección Departamental de Matemática Aplicada, Escuela de Estadística \\ Universidad Complutense de Madrid \\ 28040 Madrid, Spain \\ E-mail: luz_fernandez-c@mat.ucm.es
}

\begin{abstract}
We review several results on interpolation of Banach algebras and factorization of weakly compact homomorphisms. We also establish a new result on interpolation of multilinear operators.
\end{abstract}

1. Introduction. A celebrated result of Davis, Figiel, Johnson and Pełczyński [15] shows that any weakly compact operator between Banach spaces can be factorized through a reflexive Banach space. In 1992, Galé, Ransford and White [16] asked whether or not the corresponding result holds in the setting of Banach algebras. The answer to this question has been given recently by Blanco, Kaijser and Ransford [5], by using an interpolation method of the type of the classical real method $(\cdot, \cdot)_{\theta, q}$ which, on one hand, respects the Banach-algebra structure and, on the other hand, it produces reflexive spaces

2000 Mathematics Subject Classification: Primary 46B70, 46M35; Secondary 46H05.

Key words and phrases: real interpolation, Banach algebras, factoring weakly compact homomorphisms, multilinear operators.

Authors have been supported in part by the Spanish Ministerio de Educación y Ciencia (MTM2004-01888) and CAM-UCM (Grupo de Investigación 910348). F.C. supported also in part by RTM PHD FP6-511953.

The paper is in final form and no version of it will be published elsewhere. 
under suitable conditions. Previous results on interpolation of Banach algebras are due to Bishop [4], A. P. Calderón [7], Zafran [27] and Kaijser [19].

In this paper we work mainly with the general real method $(\cdot, \cdot)_{\Gamma}$, which is defined similarly to $(\cdot, \cdot)_{\theta, q}$ but replacing the weighted $\ell_{q}$ norm by a more general sequence lattice norm $\Gamma$ (see [25], [24] and [6]). We review a result of Astashkin [1] and Martínez and the present authors [12] which shows a necessary and sufficient condition on $\Gamma$ for $(\cdot, \cdot)_{\Gamma}$ to preserve the Banach-algebra structure. That is to say, for $\left(A_{0}, A_{1}\right)_{\Gamma}$ to be a Banach algebra provided that $\left(A_{0}, A_{1}\right)$ is a compatible couple of Banach algebras such that the two multiplications agree on $A_{0} \cap A_{1}$. That condition turns out to be that $\Gamma$ is a Banach algebra with multiplication defined as convolution. As a consequence of this result and interpolation properties of weakly compact operators (see [23] and [10]), we shall recover the result of Blanco, Kaijser and Ransford [5].

We shall also review other results of Astashkin [1] and the authors [9] on the relationship between interpolation of Banach algebras and interpolation of bilinear operators. Finally, we shall establish a new result on multilinear interpolation.

The organization of the paper is as follows. In Section 2 we describe the approach of [12] to factorization of weakly compact homomorphisms. In Section 3 we review some basic facts on the general real method $(\cdot, \cdot)_{\Gamma}$. Section 4 is devoted to interpolation of Banach algebras and, in the final Section 5, we deal with interpolation of multilinear operators.

2. Factoring weakly compact homomorphisms. Let $A$ be a Banach algebra, that is, an algebra which is also a Banach space and such that there is a constant $c_{A}>0$ such that for all $x, y \in A$ we have

$$
\|x y\|_{A} \leq c_{A}\|x\|_{A}\|y\|_{A} .
$$

Habitually it is required that $c_{A}=1$ but this definition is equivalent to the usual one (see [14], Exer. VII.1.1) and it is better for our aims.

Galé, Ransford and White asked in [16] whether or not every weakly compact homomorphism between Banach algebras $\Phi: A \rightarrow B$ factors through a reflexive Banach algebra $W$ with Banach algebra homomorphisms $\Phi_{1}, \Phi_{2}$ as factors

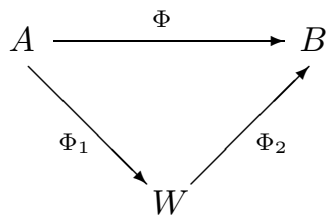

This problem is related to the well-known result of Davis, Figiel, Johnson and Pełczyński [15] on factorization of weakly compact operators through reflexive Banach spaces. It is just the corresponding result in the setting of Banach algebras.

The answer was given only recently by Blanco, Kaijser and Ransford [5]. In order to see the difficulties to establish this result, let us follow the approach given by Martínez and the present authors [12] to the result of Blanco, Kaijser and Ransford. 
Given the homomorphism $\Phi: A \rightarrow B$, let $\operatorname{Ker}(\Phi)$ be its kernel and let $F=A / \operatorname{Ker}(\Phi)$ be the quotient Banach algebra. The quotient mapping $Q(x)=[x]$ is a Banach algebra homomorphism, as well as the map $j: F \rightarrow B$ defined by $j([x])=\Phi(x)$. Moreover, the map $j$ is one-to-one. Let $W_{0}=j(Q(A))=\{\Phi(x): x \in A\}$ endowed with the norm

$$
\|\Phi(x)\|_{W_{0}}=\|[x]\|_{F}=\inf \left\{\|y\|_{A}: \Phi(x)=\Phi(y)\right\} .
$$

Then $W_{0}$ is a Banach algebra, continuously embedded in $W_{1}=B$. Multiplication in $W_{0}$ is the same as in $W_{1}$. Moreover, the open unit ball of $W_{0}$ is contained in the image by $\Phi$ of the unit ball of $A$, so the embedding from $W_{0}$ into $W_{1}$ is weakly compact. The following picture illustrates the situation:

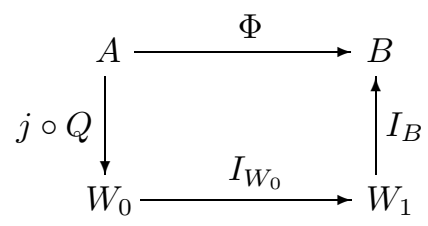

To establish the factorization result we only need to find a reflexive Banach algebra $W$ such that $W_{0} \hookrightarrow W \hookrightarrow W_{1}$, where $\hookrightarrow$ means continuous inclusion. This will yield the wanted factorization by taking $\Phi_{1}=j \circ Q=\Phi$ and $\Phi_{2}=I_{B}$.

To construct $W$ one might try to use some interpolation method. In the next two sections we study this point.

3. Real interpolation. Let $\bar{X}=\left(X_{0}, X_{1}\right)$ be a Banach couple, that is, two Banach spaces $X_{j}, j=0,1$, which are continuously embedded in some Hausdorff topological vector space. Peetre's $K$ - and J-functionals are defined by

$$
K(t, x)=K(t, x ; \bar{X})=\inf \left\{\left\|x_{0}\right\|_{X_{0}}+t\left\|x_{1}\right\|_{X_{1}}: x=x_{0}+x_{1}, x_{j} \in X_{j}\right\}, \quad x \in X_{0}+X_{1}
$$

and

$$
J(t, x)=J(t, x ; \bar{X})=\max \left\{\|x\|_{X_{0}}, t\|x\|_{X_{1}}\right\}, \quad x \in X_{0} \cap X_{1} .
$$

Notice that the pair $\left(W_{0}, W_{1}\right)$ arising in Section 2 is a Banach couple. Indeed, we can take $W_{1}$ as the Hausdorff topological vector space. This special case where $W_{0} \hookrightarrow W_{1}$ is called an ordered couple. Note that $W_{0} \cap W_{1}=W_{0}$ and $W_{0}+W_{1}=W_{1}$.

Let $\Gamma$ be a Banach lattice of real valued sequences with $\mathbb{Z}$ as index set, that is to say, whenever $\mu=\left\{\mu_{m}\right\}$ belongs to $\Gamma$ and $\xi=\left\{\xi_{m}\right\}$ satisfies $\left|\xi_{m}\right| \leq\left|\mu_{m}\right|$ for each $m \in \mathbb{Z}$, then $\xi \in \Gamma$ and $\|\xi\|_{\Gamma} \leq\|\mu\|_{\Gamma}$. We also assume that $\Gamma$ contains all sequences with only finitely many non-zero co-ordinates.

We say that $\Gamma$ is $K$-non-trivial if

$$
\left\{\min \left(1,2^{m}\right)\right\} \in \Gamma \text {. }
$$

The lattice $\Gamma$ is said to be $J$-non-trivial if

$$
\sup \left\{\sum_{m=-\infty}^{\infty} \min \left(1,2^{-m}\right)\left|\xi_{m}\right|:\left\|\left\{\xi_{m}\right\}\right\|_{\Gamma} \leq 1\right\}<\infty .
$$


These conditions are equivalent to

$$
\ell_{\infty}\left(\max \left(1,2^{-m}\right)\right) \subseteq \Gamma
$$

and

$$
\Gamma \subseteq \ell_{1}\left(\min \left(1,2^{-m}\right)\right),
$$

respectively (see [24]). Here, given any sequence $\left\{\omega_{m}\right\}$ of positive numbers and $1 \leq q \leq \infty$, we put $\ell_{q}\left(\omega_{m}\right)=\left\{\xi=\left\{\xi_{m}\right\}:\left\{\omega_{m} \xi_{m}\right\} \in \ell_{q}\right\}$.

The associated space $\Gamma^{\prime}$ of $\Gamma$ consists of all sequences $\eta=\left\{\eta_{m}\right\}$ for which

$$
\|\eta\|_{\Gamma^{\prime}}=\sup \left\{\sum_{m=-\infty}^{\infty}\left|\xi_{m} \eta_{m}\right|:\|\xi\|_{\Gamma} \leq 1\right\}<\infty .
$$

If $\xi \in \Gamma$ and $\eta \in \Gamma^{\prime}$, we put

$$
<\xi, \eta>=\sum_{m=-\infty}^{\infty} \xi_{m} \eta_{m} .
$$

Observe that if $\Gamma$ satisfies (4) then $\ell_{\infty}\left(\max \left(1,2^{m}\right)\right) \subseteq \Gamma^{\prime}$ and so if for $R \in \mathbb{N}$ we define the sequences $\delta^{(R)}=\left\{\mu_{m}\right\}, \rho^{(R)}=\left\{\eta_{m}\right\}$ by

(5) $\quad \mu_{m}=1$ if $m \leq R$ and 0 otherwise, $\quad \eta_{m}=2^{-m}$ if $m \geq R$ and 0 otherwise

then it turns out that $\delta^{(0)}$ and $\rho^{(0)}$ belongs to $\Gamma^{\prime}$.

Let $\Gamma$ be a $K$-non-trivial sequence space and let $\bar{X}=\left(X_{0}, X_{1}\right)$ be a Banach couple. The abstract $K$-space $\bar{X}_{\Gamma ; K}=\left(X_{0}, X_{1}\right)_{\Gamma ; K}$ is defined as the collection of all $x \in X_{0}+X_{1}$ such that $\left\{K\left(2^{m}, x\right)\right\} \in \Gamma$. The norm of $\bar{X}_{\Gamma ; K}$ is $\|x\|_{\bar{X}_{\Gamma ; K}}=\left\|\left\{K\left(2^{m}, x\right)\right\}\right\|_{\Gamma}$.

Let $\Gamma$ be a $J$-non-trivial sequence space. The abstract $J$-space $\bar{X}_{\Gamma ; J}=\left(X_{0}, X_{1}\right)_{\Gamma ; J}$ is formed by all those elements $x \in X_{0}+X_{1}$ for which there is a sequence $\left\{u_{m}\right\} \subseteq X_{0} \cap X_{1}$ such that

$$
\left.x=\sum_{m=-\infty}^{\infty} u_{m} \text { (convergence in } X_{0}+X_{1}\right) \text { and }\left\{J\left(2^{m}, u_{m}\right)\right\} \in \Gamma .
$$

The norm in $\bar{X}_{\Gamma ; J}=\left(X_{0}, X_{1}\right)_{\Gamma ; J}$ is

$$
\|x\|_{\bar{X}_{\Gamma ; J}}=\inf \left\{\left\|\left\{J\left(2^{m}, u_{m}\right)\right\}\right\|_{\Gamma}\right\}
$$

where the infimum is taken over all representations of $x$ satisfying (6). It turns out that $X_{0} \cap X_{1} \hookrightarrow \bar{X}_{\Gamma ; J} \hookrightarrow X_{0}+X_{1}$ and $X_{0} \cap X_{1} \hookrightarrow \bar{X}_{\Gamma ; K} \hookrightarrow X_{0}+X_{1}$.

The classical real method $(\cdot, \cdot)_{\theta, q}$ (see [3] or [26]) can be recovered by the choice $\Gamma=\ell_{q}\left(2^{-\theta m}\right)$. Namely,

$$
\left(X_{0}, X_{1}\right)_{\theta, q}=\left(X_{0}, X_{1}\right)_{\ell_{q}\left(2^{-\theta m}\right) ; K}=\left(X_{0}, X_{1}\right)_{\ell_{q}\left(2^{-\theta m}\right) ; J}
$$

with equivalence of norms. We write $\|\cdot\|_{\theta, q}$ for any of the norms in $\left(X_{0}, X_{1}\right)_{\theta, q}$.

In general $K$ - and $J$-spaces do not coincide. We only have that $\bar{X}_{\Gamma ; K} \hookrightarrow \bar{X}_{\Gamma ; J}$ for any Banach couple $\bar{X}$. The converse inclusion holds if the Calderón transform

$$
\Omega\left\{\xi_{m}\right\}=\left\{\sum_{k=-\infty}^{\infty} \min \left(1,2^{m-k}\right) \xi_{k}\right\}_{m \in \mathbb{Z}}
$$


is a bounded operator in $\Gamma$ (see [24], Lemma 2.5). If we have equality, we denote any of these two spaces by $\bar{X}_{\Gamma}=\left(X_{0}, X_{1}\right)_{\Gamma}$ and we write $\|\cdot\|_{\bar{X}_{\Gamma}}$ to mean any of the equivalent norms $\|\cdot\|_{\bar{X}_{\Gamma ; K}}$ or $\|\cdot\|_{\bar{X}_{\Gamma ; J}}$. This however will not cause any confusion.

In our later considerations, it will be important to know when one can compute the $J$-norm of the elements in $X_{0} \cap X_{1}$ by using only finite representations. This property is related with the behaviour of shift operators $\left\{\tau_{k}\right\}_{k \in \mathbb{Z}}$ on $\Gamma$. Recall that $\tau_{k}$ is defined by $\tau_{k}\left\{\xi_{m}\right\}=\left\{\xi_{m+k}\right\}_{m \in \mathbb{Z}}$. A natural assumption on shift operators is

$$
\lim _{n \rightarrow \infty} 2^{-n}\left\|\tau_{n}\right\|_{\Gamma, \Gamma}=0 \quad \text { and } \quad \lim _{n \rightarrow \infty}\left\|\tau_{-n}\right\|_{\Gamma, \Gamma}=0
$$

(see [11], [12] or [13]). Sometimes one even has

$$
\sup \left\{\left\|\tau_{-R}\right\|_{\Gamma, \Gamma}\left\|\tau_{R}\right\|_{\Gamma, \Gamma}: R \in \mathbb{N}\right\}<\infty .
$$

This is, for example, the case of the classical real method where $\Gamma=\ell_{q}\left(2^{-\theta m}\right)$ because $\left\|\tau_{R}\right\|_{\ell_{q}\left(2^{-\theta m}\right), \ell_{q}\left(2^{-\theta m}\right)} \leq 2^{\theta R}$.

We can compare the norms of shift operators in $\Gamma$ and $\Gamma^{\prime}$ as the following lemma shows.

LEMmA 3.1. Let $\Gamma$ be a lattice satisfying (2). Then, for each $k \in \mathbb{Z}$, we have $\left\|\tau_{k}\right\|_{\Gamma^{\prime}, \Gamma^{\prime}} \leq$ $\left\|\tau_{-k}\right\|_{\Gamma, \Gamma}$.

Proof. According to the definition of the norm in $\Gamma^{\prime}$ we obtain

$$
\begin{aligned}
\left\|\tau_{k}\right\|_{\Gamma^{\prime}, \Gamma^{\prime}} & =\sup \left\{\left\|\tau_{k} \eta\right\|:\|\eta\|_{\Gamma^{\prime}} \leq 1\right\}=\sup \left\{\left|<\xi, \tau_{k} \eta>\right|:\|\eta\|_{\Gamma^{\prime}} \leq 1,\|\xi\|_{\Gamma} \leq 1\right\} \\
& =\sup \left\{\left|<\tau_{-k} \xi, \eta>\right|:\|\eta\|_{\Gamma^{\prime}} \leq 1,\|\xi\|_{\Gamma} \leq 1\right\} \\
& \leq \sup \left\{\left\|\tau_{-k} \xi\right\|_{\Gamma}\|\eta\|_{\Gamma^{\prime}}:\|\eta\|_{\Gamma^{\prime}} \leq 1,\|\xi\|_{\Gamma} \leq 1\right\}=\left\|\tau_{-k}\right\|_{\Gamma, \Gamma} .
\end{aligned}
$$

Let $e_{m}$ be the sequence which is zero at all co-ordinates but the $m$ th co-ordinate where it is 1 . Next we show a first result on the equivalence between the norms $\|x\|_{\bar{X}_{\Gamma ; J}}$ and

$$
\|x\|_{\bar{X}_{\Gamma ; J}}^{*}=\inf \left\{\left\|\left\{J\left(2^{m}, u_{m}\right)\right\}\right\|_{\Gamma}: x=\sum_{m=-\infty}^{\infty} u_{m} \text { and only a finite number of } u_{m} \neq 0\right\}
$$

in $X_{0} \cap X_{1}$. In the case of the classical real method, this result was proved by Janson in [18], Lemma 1.

LEMMA 3.2. Let $\Gamma$ be a lattice satisfying (2). Assume also that shift operators on $\Gamma$ satisfy (7) and (8). Then, for any Banach couple $\bar{X}=\left(X_{0}, X_{1}\right)$, the norms $\|\cdot\|_{\bar{X}_{\Gamma ; J}}$ and $\|\cdot\|_{\bar{X}_{\Gamma ; J}}^{*}$ are equivalent on $X_{0} \cap X_{1}$.

Proof. Clearly $\|x\|_{\bar{X}_{\Gamma ; J}} \leq\|x\|_{\bar{X}_{\Gamma ; J}}^{*}$ for any $x \in X_{0} \cap X_{1}$. Let us check that the converse inequality holds with a constant independent of $x$. Take any $x \in X_{0} \cap X_{1}$ and any $J$-representation $x=\sum_{m=-\infty}^{\infty} u_{m}$ of $x$ with $\left\|\left\{J\left(2^{m}, u_{m}\right)\right\}\right\|_{\Gamma} \leq 2\|x\|_{\bar{X}_{\Gamma ; J}}$. Using (7) we can find $R \in \mathbb{N}$ such that

$$
\max \left(2^{-R}\left\|\tau_{R}\right\|_{\Gamma, \Gamma}\left\|e_{0}\right\|_{\Gamma}\|x\|_{X_{1}},\left\|\tau_{-R}\right\|_{\Gamma, \Gamma}\left\|e_{0}\right\|_{\Gamma}\|x\|_{X_{0}}\right) \leq\|x\|_{\bar{X}_{\Gamma ; J}} .
$$

Put $x_{R}=\sum_{m \geq R} u_{m}$ and $x_{-R}=\sum_{m \leq-R} u_{m}$. We claim that $x_{R}$ and $x_{-R}$ belong to $X_{0} \cap X_{1}$. In order to check it, we shall use the sequences $\delta^{(R)}, \rho^{(R)}$ defined in (5). We 
have

$$
\begin{aligned}
\left\|x_{R}\right\|_{X_{0}} & \leq\|x\|_{X_{0}}+\sum_{m \leq R-1}\left\|u_{m}\right\|_{X_{0}} \leq\|x\|_{X_{0}}+\sum_{m \leq R-1} J\left(2^{m}, u_{m}\right) \\
& \leq\|x\|_{X_{0}}+\left\|\left\{J\left(2^{m}, u_{m}\right)\right\}\right\|_{\Gamma}\left\|\delta^{(R-1)}\right\|_{\Gamma^{\prime}} \\
& =\|x\|_{X_{0}}+2\|x\|_{\bar{X}_{\Gamma ; J}}\left\|\tau_{-(R-1)}\right\|_{\Gamma^{\prime}, \Gamma^{\prime}}\left\|\delta^{(0)}\right\|_{\Gamma^{\prime}} \leq\|x\|_{X_{0}}+2 c_{1}\left\|\tau_{R-1}\right\|_{\Gamma, \Gamma}\|x\|_{\bar{X}_{\Gamma ; J}}
\end{aligned}
$$

where we have used Lemma 3.1 in the last inequality. As for the norm in $X_{1}$ we get

$$
\begin{aligned}
\left\|x_{R}\right\|_{X_{1}} & \leq \sum_{m \geq R}\left\|u_{m}\right\|_{X_{1}} \leq \sum_{m \geq R} 2^{-m} J\left(2^{m}, u_{m}\right) \leq\left\|\left\{J\left(2^{m}, u_{m}\right)\right\}\right\|_{\Gamma}\left\|\rho^{(R)}\right\|_{\Gamma^{\prime}} \\
& \leq 2\|x\|_{\bar{X}_{\Gamma ; J}} 2^{-N}\left\|\tau_{-R} \rho^{(0)}\right\|_{\Gamma^{\prime}} \leq 2 c_{2} 2^{-R}\left\|\tau_{R}\right\|_{\Gamma, \Gamma}\|x\|_{\bar{X}_{\Gamma ; J}} .
\end{aligned}
$$

Therefore, for some constant $c_{3}$ we obtain

$$
J\left(2^{R}, x_{R}\right) \leq\|x\|_{X_{0}}+c_{3}\left\|\tau_{R}\right\|_{\Gamma, \Gamma}\|x\|_{\bar{X}_{\Gamma ; J}} .
$$

Similarly,

$$
J\left(2^{-R}, x_{-R}\right) \leq 2^{-R}\|x\|_{X_{1}}+c_{3}\left\|\tau_{-R}\right\|_{\Gamma, \Gamma}\|x\|_{\bar{X}_{\Gamma ; J}} .
$$

Consequently, we have for $x$ the finite representation $x=x_{-R}+\sum_{m=-R+1}^{R-1} x_{m}+x_{R}$ and so

$$
\begin{aligned}
\|x\|_{\bar{X}_{\Gamma ; J}}^{*} & \leq\left\|J\left(2^{-R}, x_{-R}\right) e_{-R}\right\|_{\Gamma}+\left\|\left\{J\left(2^{m}, x_{m}\right)\right\}\right\|_{\Gamma}+\left\|J\left(2^{R}, x_{R}\right) e_{R}\right\|_{\Gamma} \\
& \leq J\left(2^{-R}, x_{-R}\right)\left\|\tau_{R}\right\|_{\Gamma, \Gamma}\left\|e_{0}\right\|_{\Gamma}+2\|x\|_{\bar{X}_{\Gamma ; J}}+J\left(2^{R}, x_{R}\right)\left\|\tau_{-R}\right\|_{\Gamma, \Gamma}\left\|e_{0}\right\|_{\Gamma} \\
& \leq\left\|e_{0}\right\|_{\Gamma}\left(2^{-R}\left\|\tau_{R}\right\|_{\Gamma, \Gamma}\|x\|_{X_{1}}+\left\|\tau_{-R}\right\|_{\Gamma, \Gamma}\|x\|_{X_{0}}+c_{4}\|x\|_{\bar{X}_{\Gamma ; J}}\right)+2\|x\|_{\bar{X}_{\Gamma ; J}} \\
& \leq c_{5}\|x\|_{\bar{X}_{\Gamma ; J}}
\end{aligned}
$$

where the last inequality follows from (9). This yields that the norms $\|\cdot\|_{\bar{X}_{\Gamma ; J}}$ and $\|\cdot\|_{\bar{X}_{\Gamma ; J}}^{*}$ are equivalent on $X_{0} \cap X_{1}$ and completes the proof.

Assumption (8) is not suitable for our latter considerations, but equivalence between $\|\cdot\|_{\bar{X}_{\Gamma ; J}}$ and $\|\cdot\|_{\bar{X}_{\Gamma ; J}}^{*}$ on $X_{0} \cap X_{1}$ still holds if we replace (8) by boundedness of the Calderón transform $\Omega$ in $\Gamma$ as was shown in [12], Lemma 2.2

LEMma 3.3. Let $\Gamma$ be a $K$ - and $J$-non-trivial lattice satisfying (7) and with $\Omega$ bounded in $\Gamma$. Then for any Banach couple $\bar{X}=\left(X_{0}, X_{1}\right)$ and for any $x \in X_{0} \cap X_{1}$ we have $\|x\|_{\bar{X}_{\Gamma ; J}}^{*} \leq 8\|x\|_{\bar{X}_{\Gamma ; K}}$.

Note that under the assumptions of Lemma 3.3 we have $\bar{X}_{\Gamma ; K}=\bar{X}_{\Gamma ; J}$.

It will be important latter that $X_{0} \cap X_{1}$ is dense in $\bar{X}_{\Gamma}$. It is not hard to check that this happens if

$$
\xi=\lim _{n \rightarrow \infty} \sum_{j=-n}^{n} \xi_{j} e_{j} \quad(\text { convergence in } \Gamma) \text { for any } \xi=\left\{\xi_{m}\right\} \in \Gamma .
$$

For example, this is the case of $\left(X_{0}, X_{1}\right)_{\theta, q}$ if $q<\infty$.

4. Interpolation of Banach algebras. By a couple of Banach algebras $\bar{A}=\left(A_{0}, A_{1}\right)$ we understand a Banach couple formed by Banach algebras $A_{j}, j=0,1$, such that multiplications in $A_{0}$ and $A_{1}$ coincide in $A_{0} \cap A_{1}$. 
Note that the ordered couple $\left(W_{0}, W_{1}\right)$ which arose in Section 2 is a couple of Banach algebras.

Definition 4.1. Let $\Gamma$ be a $K$ - and $J$-non-trivial sequence space satisfying (7) and (10), and with $\Omega$ bounded in $\Gamma$. We say that the method $(\cdot, \cdot)_{\Gamma}$ preserves the Banach-algebra structure if given any couple of Banach algebras $\bar{A}=\left(A_{0}, A_{1}\right)$ there is a constant $c_{\bar{A}_{\Gamma}}>0$ such that for all $x, y \in A_{0} \cap A_{1}$

$$
\|x y\|_{\bar{A}_{\Gamma}} \leq c_{\bar{A}_{\Gamma}}\|x\|_{\bar{A}_{\Gamma}}\|y\|_{\bar{A}_{\Gamma}} .
$$

Notice that multiplication of $x, y$ is well defined because these vectors belong to $A_{0} \cap A_{1}$ and multiplications in $A_{0}$ and $A_{1}$ agree on $A_{0} \cap A_{1}$.

Observe also that, since $A_{0} \cap A_{1}$ is dense in $\left(A_{0}, A_{1}\right)_{\Gamma}$, multiplication can be extended by continuity to the whole of $\left(A_{0}, A_{1}\right)_{\Gamma}$, turning $\left(A_{0}, A_{1}\right)_{\Gamma}$ into a Banach algebra.

The following result was essentially established by Bishop [4]. So far as we are aware, this is the first result known on an interpolation method that preserves Banach algebras.

THEOREM 4.2. Let $0<\theta<1$. The classical real method $(\cdot, \cdot)_{\theta, 1}$ with parameters $(\theta, 1)$ preserves the Banach-algebra structure.

Proof. We start by showing that for any Banach couple $\bar{X}=\left(X_{0}, X_{1}\right)$ the norm $\|\cdot\|_{\theta, 1}^{*}$ is equivalent to

$$
\|x\|_{\theta, 1}^{\diamond}=\inf \left\{\sum_{m=-R}^{R}\left\|x_{m}\right\|_{X_{0}}^{1-\theta}\left\|x_{m}\right\|_{X_{1}}^{\theta}: x=\sum_{m=-R}^{R} x_{m},\left\{x_{m}\right\} \subseteq X_{0} \cap X_{1}, R \in \mathbb{N}\right\}
$$

on $X_{0} \cap X_{1}$.

Indeed, as $\|x\|_{\theta, 1} \leq c\|x\|_{X_{0}}^{1-\theta}\|x\|_{X_{1}}^{\theta}$ for any $x \in X_{0} \cap X_{1}$ (see, for example, [3] or [26]), given any finite representation $x=\sum_{m=-R}^{R} x_{m}$ we have

$$
\|x\|_{\theta, 1} \leq \sum_{m=-R}^{R}\left\|x_{m}\right\|_{\theta, 1} \leq c \sum_{m=-R}^{R}\left\|x_{m}\right\|_{X_{0}}^{1-\theta}\left\|x_{m}\right\|_{X_{1}}^{\theta} .
$$

This yields that $\|x\|_{\theta, 1}^{*} \leq c_{1}\|x\|_{\theta, 1}^{\diamond}$ for all $x \in X_{0} \cap X_{1}$. Conversely, given any $\varepsilon>0$, we can find a finite representation $x=\sum_{m=-R}^{R} x_{m}$ with $\sum_{m=-R}^{R} 2^{\theta m} J\left(2^{m}, x_{m}\right) \leq(1+\varepsilon)\|x\|_{\theta, 1}^{*}$. Whence,

$$
\begin{aligned}
\|x\|_{\theta, 1}^{\diamond} & \leq \sum_{m=-R}^{R}\left\|x_{m}\right\|_{X_{0}}^{1-\theta}\left\|x_{m}\right\|_{X_{1}}^{\theta} \leq \sum_{m=-R}^{R} J\left(2^{m}, x_{m}\right)^{1-\theta}\left(2^{-m} J\left(2^{m}, x_{m}\right)\right)^{\theta} \\
& =\sum_{m=-R}^{R} 2^{-\theta m} J\left(2^{m}, x_{m}\right) \leq(1+\varepsilon)\|x\|_{\theta, 1}^{*} .
\end{aligned}
$$

This gives the equivalence of norms on $X_{0} \cap X_{1}$.

Next take any couple of Banach algebras $\left(A_{0}, A_{1}\right)$ and any $x, y \in A_{0} \cap A_{1}$. Given any $\varepsilon>0$, there are finite representations $x=\sum_{m=-R}^{R} u_{m}, y=\sum_{k=-R}^{R} v_{k}$ such that

$$
\sum_{m=-R}^{R}\left\|u_{m}\right\|_{A_{0}}^{1-\theta}\left\|u_{m}\right\|_{A_{1}}^{\theta} \leq(1+\varepsilon)\|x\|_{\theta, 1}^{\diamond}
$$


and

$$
\sum_{k=-R}^{R}\left\|v_{k}\right\|_{A_{0}}^{1-\theta}\left\|v_{k}\right\|_{A_{1}}^{\theta} \leq(1+\varepsilon)\|y\|_{\theta, 1}^{\diamond} .
$$

Then $x y=\sum_{m, k=-R}^{R} u_{m} v_{k}$ and so

$$
\begin{aligned}
\|x y\|_{\theta, 1}^{\diamond} & \leq \sum_{m, k=-R}^{R}\left\|u_{m} v_{k}\right\|_{A_{0}}^{1-\theta}\left\|u_{m} v_{k}\right\|_{A_{1}}^{\theta} \\
& \leq \max \left(c_{A_{0}}, c_{A_{1}}\right) \sum_{m, k=-R}^{R}\left\|u_{m}\right\|_{A_{0}}^{1-\theta}\left\|u_{m}\right\|_{A_{1}}^{\theta}\left\|v_{k}\right\|_{A_{0}}^{1-\theta}\left\|v_{k}\right\|_{A_{1}}^{\theta} \\
& \leq \max \left(c_{A_{0}}, c_{A_{1}}\right)\left(\sum_{m=-R}^{R}\left\|u_{m}\right\|_{A_{0}}^{1-\theta}\left\|u_{m}\right\|_{A_{1}}^{\theta}\right)\left(\sum_{k=-R}^{R}\left\|v_{k}\right\|_{A_{0}}^{1-\theta}\left\|v_{k}\right\|_{A_{1}}^{\theta}\right) \\
& \leq \max \left(c_{A_{0}}, c_{A_{1}}\right)(1+\varepsilon)^{2}\|x\|_{\theta, 1}^{\diamond}\|y\|_{\theta, 1}^{\diamond} .
\end{aligned}
$$

Letting $\varepsilon \rightarrow 0$, we see that $\left(A_{0}, A_{1}\right)_{\theta, 1}$ is a Banach algebra.

If in the definition of $(\cdot, \cdot)_{\theta, 1}$ we replace the function $t^{\theta}$ by a more general function parameter $f(t)$ then we get the more general method $(\cdot, \cdot)_{f, 1}=(\cdot, \cdot)_{\ell_{1}\left(1 / f\left(2^{m}\right)\right)}$ which also preserves Banach algebras as was established by Zafran [27] and Kaijser [19].

As for the complex interpolation method $(\cdot, \cdot)_{[\theta]}$, it was proved by A.P. Calderón in his famous seminal paper [7] that it preserves the Banach-algebra structure.

In principle we might try to apply to the ordered couple of Banach algebras $\left(W_{0}, W_{1}\right)$ any of these interpolation methods to complete the proof of the factorization theorem following the approach described in Section 2. The outcome is going to be a Banach algebra $E$ such that $W_{0} \hookrightarrow E \hookrightarrow W_{1}$. But we want $E$ to be reflexive and to obtain it we can use that the embedding $W_{0} \hookrightarrow W_{1}$ is weakly compact. So we want to use an interpolation method $\mathcal{F}$ that in addition to the property of interpolating Banach algebras, it fulfils that whenever an ordered Banach couple $\left(X_{0}, X_{1}\right)$ satisfies that the embedding $X_{0} \hookrightarrow X_{1}$ is weakly compact then $\mathcal{F}\left(X_{0}, X_{1}\right)$ is reflexive. Unfortunately, no one of the methods $(\cdot, \cdot)_{\theta, 1},(\cdot, \cdot)_{f, 1},(\cdot, \cdot)_{[\theta]}$ has this last property. The following example was given by Maligranda in [21]. It shows the failure of the property for the complex method.

EXAMPLE 4.3. Let us work on $[0,1]$ with the Lebesgue measure and for $1<p<\infty$ put

$$
L_{p, \infty}=\left\{f:\|f\|_{p, \infty}=\sup \left\{t^{\frac{1}{p}-1} \int_{0}^{t} f^{*}(s) d s\right\}<\infty\right\} .
$$

Here $f^{*}$ is the non-increasing rearrangement of $f$,

$$
f^{*}(s)=\inf \{t>0: \operatorname{mes}\{w:|f(w)|>t\} \leq s\} .
$$

Let $L_{p, \infty}^{0}$ be the closure of $L_{\infty}$ in $L_{p, \infty}$. The space $L_{p, \infty}^{0}$ is not reflexive because $\left(L_{p, \infty}^{0}\right)^{* *}=$ $L_{p, \infty}$.

Take $1<p<r<q<\infty$ and let $0<\theta<1$ such that $1 / r=(1-\theta) / q+\theta / p$. Then $L_{q, \infty}^{0} \hookrightarrow L_{r} \hookrightarrow L_{p, \infty}^{0}$, so the embedding $L_{q, \infty}^{0} \hookrightarrow L_{p, \infty}^{0}$ is weakly compact because $L_{r}$ is reflexive. However, applying the complex method we obtain $\left(L_{q, \infty}^{0}, L_{p, \infty}^{0}\right)_{[\theta]}=L_{r, \infty}^{0}$ which is not reflexive. 
Interpolation methods $(\cdot, \cdot)_{\theta, 1}$ and $(\cdot, \cdot)_{f, 1}$ have a worse behaviour. They do not generate reflexive spaces in general. In fact, Levy [20] proved that if $X_{0} \cap X_{1}$ is not closed in $X_{0}+X_{1}$, which is usually the case, then $\left(X_{0}, X_{1}\right)_{\theta, 1}$ contains a subspace isomorphic to $\ell_{1}$, so $\left(X_{0}, X_{1}\right)_{\theta, 1}$ cannot by reflexive. The same happens for $(\cdot, \cdot)_{f, 1}$ (see [8]).

If $1<q<\infty$ the classical real method behaves well concerning weak compactness. Indeed, Beauzamy [2] proved that $\left(A_{0}, A_{1}\right)_{\theta, q}$ is reflexive if the embedding $A_{0} \cap A_{1} \hookrightarrow$ $A_{0}+A_{1}$ is weakly compact. This results has been extended in several directions (see, for example, the paper by Heinrich [17] and by Maligranda and Quevedo [22]). But $(\cdot, \cdot)_{\theta, q}$ does not preserve the Banach-algebra structure.

In the paper by Blanco, Kaijser and Ransford [5] it is shown a class of real interpolation methods which interpolate Banach algebras and give reflexive spaces.

Next we review the behaviour of these properties for the general real method $(\cdot, \cdot)_{\Gamma}$. Results on weak compactness and the $K$-method $(\cdot, \cdot)_{\Gamma ; K}$ are due to Aizenstein and Brudny̌̌ (see [6], Thm. 4.6.8) and Mastyło [23]. The case of the $J$-method $(\cdot, \cdot)_{\Gamma ; J}$ has been studied by Manzano, Martínez and the present authors in [10]. We can summarize all these results in the following theorem.

THEOREM 4.4. Let $\Gamma$ be a reflexive lattice satisfying (7). Let $\bar{X}=\left(X_{0}, X_{1}\right)$ and $\bar{Y}=$ $\left(Y_{0}, Y_{1}\right)$ be Banach couples, and let $T \in \mathcal{L}\left(X_{0}+X_{1}, Y_{0}+Y_{1}\right)$ be a linear operator whose restrictions $T: X_{j} \rightarrow Y_{j}$ are bounded, $j=0,1$, and $T: X_{0} \cap X_{1} \rightarrow Y_{0}+Y_{1}$ is weakly compact.

(i) If $\Gamma$ is K-non-trivial, then $T: \bar{X}_{\Gamma ; K} \rightarrow \bar{Y}_{\Gamma ; K}$ is weakly compact.

(ii) If $\Gamma$ is J-non-trivial, then $T: \bar{X}_{\Gamma ; J} \rightarrow \bar{Y}_{\Gamma ; J}$ is weakly compact.

As for interpolation of Banach algebras, the result by Astashkin [1] and by Martínez and the authors [12] reads as follows.

THEOREM 4.5. Let $\Gamma$ be a $K$ - and J-non-trivial lattice, with the Calderón transform bounded in $\Gamma$. Suppose that (10) is satisfied and that shift operators on $\Gamma$ fulfill (7). Then a necessary and sufficient condition for the method $(\cdot, \cdot)_{\Gamma}$ to preserve the Banach-algebra structure is that $\Gamma$ be a Banach algebra with multiplication defined as convolution

$$
\xi * \eta=\left\{\sum_{k=-\infty}^{\infty} \xi_{k} \eta_{m-k}\right\}_{m \in \mathbb{Z}}, \quad \xi=\left\{\xi_{m}\right\}, \eta=\left\{\eta_{m}\right\} .
$$

For $0<\theta<1,1<q<\infty$ and $\gamma>(q-1) / q$, one can check that the lattice $\Gamma=\ell_{q}\left(2^{-\theta m}(1+|m|)^{\gamma}\right)$ is a Banach algebra with convolution and it is also reflexive. Hence, interpolating the ordered couple of Banach algebras $\left(W_{0}, W_{1}\right)$ of Section 2 by the general real method defined by $\Gamma$, we obtain the desired reflexive Banach algebras $W=\left(W_{0}, W_{1}\right)_{\Gamma}$ that completes the proof of the factorization theorem for weakly compact homomorphisms.

The above considerations also show that the real method is not enough to complete the proof. Namely, as an immediate consequence of Theorem 4.5 we have the following characterization.

COROLlaRY 4.6. The classical real method $(\cdot, \cdot)_{\theta, q}$ preserves the Banach-algebra structure only if $q=1$. 
5. Multilinear interpolation. By means of a direct argument, we have proved in Theorem 4.2 that $(\cdot, \cdot)_{\theta, 1}$ interpolates Banach algebras. For $(\cdot, \cdot)_{f, 1}$ (see [27], [19]), for the general real method $(\cdot, \cdot)_{\Gamma}$ (see [12]) and for the methods studied in [5] the arguments are also direct. However, for the case of the complex method, the approach followed by Calderón in [7] was to prove first a multilinear interpolation theorem and then, as an immediate consequence of the bilinear result, to derive that the complex method preserves the Banach-algebra structure. It is then natural to investigate the relationship between real interpolation of Banach algebras and interpolation of bilinear operators. This problem has been considered in [1] and [9]. Next we recall their main result. We need the following definition.

Definition 5.1. Let $\Gamma$ be a $K$-and $J$-non-trivial lattice satisfying (10). If for any Banach couples $\bar{X}=\left(X_{0}, X_{1}\right), \bar{Y}=\left(Y_{0}, Y_{1}\right), \bar{V}=\left(V_{0}, V_{1}\right)$ and for any bilinear operator $T$ defined in $\left(X_{0} \cap X_{1}\right) \times\left(Y_{0} \cap Y_{1}\right)$ with values in $V_{0} \cap V_{1}$ and such that

$$
\|T(x, y)\|_{V_{j}} \leq M_{j}\|x\|_{X_{j}}\|y\|_{Y_{j}}, j=0,1 \text {, for all } x \in X_{0} \cap X_{1}, y \in Y_{0} \cap Y_{1},
$$

there exists a constant $M=M(T)$ such that

$$
\|T(x, y)\|_{\bar{V}_{\Gamma ; K}} \leq M\|x\|_{\bar{X}_{\Gamma ; J}}\|y\|_{\bar{Y}_{\Gamma ; J}} \text { for all } x \in X_{0} \cap X_{1}, y \in Y_{0} \cap Y_{1}
$$

then we say that the bilinear interpolation theorem $J_{\Gamma} \times J_{\Gamma} \rightarrow K_{\Gamma}$ holds.

We say that the bilinear interpolation theorem $J_{\Gamma} \times J_{\Gamma} \rightarrow J_{\Gamma}$ is fulfilled if a similar condition is satisfied but replacing $\bar{V}_{\Gamma ; K}$ by $\bar{V}_{\Gamma ; J}$.

By density of $X_{0} \cap X_{1}$ in $\bar{X}_{\Gamma ; J}$ and $Y_{0} \cap Y_{1}$ in $\bar{Y}_{\Gamma ; J}$, if the theorem $J_{\Gamma} \times J_{\Gamma} \rightarrow K_{\Gamma}$ holds then $T$ can be uniquely extended to a continuous bilinear mapping from $\bar{X}_{\Gamma ; J} \times \bar{Y}_{\Gamma ; J}$ to $\bar{V}_{\Gamma ; K}$. For the case of the theorem $J_{\Gamma} \times J_{\Gamma} \rightarrow J_{\Gamma}$, the extension is from $\bar{X}_{\Gamma ; J} \times \bar{Y}_{\Gamma ; J}$ to $\bar{V}_{\Gamma ; J}$.

Since $\bar{V}_{\Gamma ; K} \hookrightarrow \bar{V}_{\Gamma ; J}$, it is clear that if the theorem $J_{\Gamma} \times J_{\Gamma} \rightarrow K_{\Gamma}$ holds, then the theorem $J_{\Gamma} \times J_{\Gamma} \rightarrow J_{\Gamma}$ is also fulfilled.

The following result is proved in [9].

THEOREM 5.2. Let $\Gamma$ be a $K$ - and J-non-trivial lattice satisfying (10). Assume also that shift operators in $\Gamma$ fulfil (7). Then the following conditions are equivalent.

(i) The bilinear interpolation theorem $J_{\Gamma} \times J_{\Gamma} \rightarrow K_{\Gamma}$ holds.

(ii) $\Gamma$ is a Banach algebra with multiplication defined as convolution.

(iii) The Calderón transform $\Omega$ is bounded in $\Gamma$ and the theorem $J_{\Gamma} \times J_{\Gamma} \rightarrow J_{\Gamma}$ is satisfied.

(iv) $\Omega$ is bounded in $\Gamma$ and $(\cdot, \cdot)_{\Gamma}$ preserves the Banach-algebra structure.

We finish the paper with a new multilinear result.

THEOREM 5.3. Let $\Gamma_{0}, \Gamma_{1}, \ldots, \Gamma_{n}$ be $K$ - and J-non-trivial lattices satisfying (7), (10) and with $\Omega$ bounded in $\Gamma_{k}$ for $k=0, \ldots, n$. Assume also that the operator $\mathcal{R}$ defined by

$$
\mathcal{R}\left(\xi^{(1)}, \ldots, \xi^{(n)}\right)=\left\{\sum_{\sum_{\nu=1}^{n} k_{\nu}=m}\left(\prod_{\nu=1}^{n} \xi_{k_{\nu}}^{(\nu)}\right)\right\}_{m \in \mathbb{Z}}, \quad \xi^{(\nu)}=\left\{\xi_{m}^{(\nu)}\right\} \in \Gamma_{\nu}
$$


is bounded from $\prod_{\nu=1}^{n} \Gamma_{\nu}$ to $\Gamma_{0}$, that is, for some constant $M$

$$
\left\|\mathcal{R}\left(\xi^{(1)}, \ldots, \xi^{(n)}\right)\right\|_{\Gamma_{0}} \leq M \prod_{\nu=1}^{n}\left\|\xi^{(\nu)}\right\|_{\Gamma_{\nu}}, \quad \xi^{(\nu)} \in \Gamma_{\nu} .
$$

Let $\bar{X}^{(\nu)}=\left(X_{0}^{(\nu)}, X_{1}^{(\nu)}\right), \nu=1, \ldots, n$, and $\bar{Y}=\left(Y_{0}, Y_{1}\right)$ be Banach couples and let $T$ : $\prod_{\nu=1}^{n}\left(X_{0}^{(\nu)} \cap X_{1}^{(\nu)}\right) \rightarrow Y_{0} \cap Y_{1}$ be a multilinear operator such that for any $x_{\nu} \in X_{0}^{(\nu)} \cap X_{1}^{(\nu)}$,

$$
\left\|T\left(x_{1}, \ldots, x_{n}\right)\right\|_{Y_{j}} \leq M_{j} \prod_{\nu=1}^{n}\left\|x_{\nu}\right\|_{X_{j}^{(\nu)}}, j=0,1 .
$$

Then there is a constant $D$ such that

$$
\left\|T\left(x_{1}, \ldots, x_{n}\right)\right\|_{\bar{Y}_{\Gamma_{0}}} \leq D \prod_{\nu=1}^{n}\left\|x_{\nu}\right\|_{\bar{X}_{\Gamma_{\nu}}^{(\nu)}}
$$

and thus $T$ may be uniquely extended to a bounded multilinear mapping from $\prod_{\nu=1}^{n} \bar{X}_{\Gamma_{\nu}}^{(\nu)}$ to $\bar{Y}_{\Gamma_{0}}$.

Proof. Take any $x_{\nu} \in X_{0}^{(\nu)} \cap X_{1}^{(\nu)}, \nu=1, \ldots, n$, and let $x_{\nu}=\sum_{m=-\infty}^{\infty} u_{m}^{(\nu)}$ be any $J$-representation with only a finite number of terms $u_{m}^{(\nu)}$ distinct from zero. For $m \in \mathbb{Z}$, put

Then $w_{m} \in Y_{0} \cap Y_{1}$ and

$$
w_{m}=\sum_{\sum_{\nu=1}^{n} k_{\nu}=m} T\left(u_{k_{1}}^{(1)}, \ldots, u_{k_{n}}^{(n)}\right) .
$$

$$
T\left(x_{1}, \ldots, x_{n}\right)=\sum_{m}\left(\sum_{\sum_{\nu=1}^{n} k_{\nu}=m} T\left(u_{k_{1}}^{(1)}, \ldots, u_{k_{n}}^{(n)}\right)\right)=\sum_{m} w_{m} .
$$

Moreover

$$
\begin{aligned}
J\left(2^{m}, w_{m}\right) & \leq \sum_{\sum_{\nu=1}^{n} k_{\nu}=m} \max \left(M_{0} \prod_{\nu=1}^{n}\left\|u_{k_{\nu}}^{(\nu)}\right\|_{X_{0}^{(\nu)}}, 2^{m} M_{1} \prod_{\nu=1}^{n}\left\|u_{k_{\nu}}^{(\nu)}\right\|_{X_{1}^{(\nu)}}\right) \\
& \leq \max \left(M_{0}, M_{1}\right) \sum_{\sum_{\nu=1}^{n} k_{\nu}=m}\left(\prod_{\nu=1}^{n} J\left(2^{k_{\nu}}, u_{k_{\nu}}^{(\nu)} ; X_{0}^{(\nu)}, X_{1}^{(\nu)}\right)\right) .
\end{aligned}
$$

Consequently,

$$
\begin{aligned}
& \left\|T\left(x_{1}, \ldots, x_{n}\right)\right\|_{\bar{Y}_{\Gamma_{0}}} \leq\left\|\left\{J\left(2^{m}, w_{m}\right)\right\}\right\|_{\Gamma_{0}} \\
& \leq \max \left(M_{0}, M_{1}\right)\left\|\left\{\sum_{\sum_{\nu=1}^{n} k_{\nu}=m}\left(\prod_{\nu=1}^{n} J\left(2^{k_{\nu}}, u_{k_{\nu}}^{(\nu)} ; X_{0}^{(\nu)}, X_{1}^{(\nu)}\right)\right)\right\}\right\|_{\Gamma_{0}} \\
& =\max \left(M_{0}, M_{1}\right)\left\|\mathcal{R}\left(\left\{J\left(2^{m}, u_{m}^{(1)} ; X_{0}^{(1)}, X_{1}^{(1)}\right)\right\}_{m}, \ldots,\left\{J\left(2^{m}, u_{m}^{(n)} ; X_{0}^{(n)}, X_{1}^{(n)}\right)\right\}_{m}\right)\right\|_{\Gamma_{0}} \\
& \leq M \max \left(M_{0}, M_{1}\right) \prod_{\nu=1}^{n}\left\|\left\{J\left(2^{m}, u_{m}^{(\nu)} ; X_{0}^{(\nu)}, X_{1}^{(\nu)}\right)\right\}\right\|_{\Gamma_{\nu}} .
\end{aligned}
$$

By Lemma 3.3, this yields that for some constant $D$ we have that

$$
\left\|T\left(x_{1}, \ldots, x_{n}\right)\right\|_{\bar{Y}_{\Gamma_{0}}} \leq D \prod_{\nu=1}^{n}\left\|x_{\nu}\right\|_{\bar{X}_{\Gamma_{\nu}}^{(\nu)}} \quad \text { for all } x_{\nu} \in X_{0}^{(\nu)} \cap X_{1}^{(\nu)}, \nu=1, \ldots, n .
$$

Since $X_{0}^{(\nu)} \cap X_{1}^{(\nu)}$ is dense in $\bar{X}_{\Gamma_{\nu}}^{(\nu)}, 1 \leq \nu \leq n$, the desired conclusion follows. 
In Theorem 5.3 it is assumed a certain property on the lattices $\Gamma_{j}$, namely that $\mathcal{R}$ is bounded, and the idea of the proof is to transfer this property to the interpolated operator by using Lemma 3.3 and the information about $T$ acting on the $n$-tuples. Similar techniques have been used to establish other multilinear theorems (see, for example, [18] and $[1])$.

Acknowledgements. It is a pleasure to thank Sten Kaijser for drawing our attention to Theorem 5.3.

\section{References}

[1] S. V. Astashkin, Interpolation of bilinear operators by the real method, Math. Notes 52 (1992), 641-648.

[2] B. Beauzamy, Espaces d'interpolation réels: topologie et géométrie, Lect. Notes in Math. 666, Springer, Berlin 1978.

[3] J. Bergh and J. Löfström, Interpolation Spaces. An Introduction, Springer, Berlin, 1976.

[4] E. A. Bishop, Holomorphic completion, analytic continuation, and the interpolation of seminorms, Ann. Math. 78 (1963), 468-500.

[5] A. Blanco, S. Kaijser and T.J. Ransford, Real interpolation of Banach algebras and factorization of weakly compact homomorphisms, J. Funct. Anal. 217 (2004), 126-141.

[6] Y. Brudnyı̆ and N. Krugljak, Interpolation Functors and Interpolation Spaces, Vol. 1, North-Holland, Amsterdam 1991.

[7] A. P. Calderón, Intermediate spaces and interpolation, the complex method, Studia Math. 24 (1964), 113-190.

[8] F. Cobos, Some spaces in which martingale difference sequences are unconditional, Bull. Polish Acad. Sciences Math. 34 (1986), 695-703.

[9] F. Cobos and L. M. Fernández-Cabrera, On the relationship between interpolation of Banach algebras and interpolation of bilinear operators, Canad. Math. Bull. (to appear).

[10] F. Cobos, L. M. Fernández-Cabrera, A. Manzano and A. Martínez, Real interpolation and closed operator ideals, J. Math. Pures Appl. 83 (2004), 417-423.

[11] F. Cobos, L. M. Fernández-Cabrera and A. Martínez, Compact operators between K- and J-spaces, Studia Math. 166 (2005), 199-220.

[12] F. Cobos, L. M. Fernández-Cabrera and A. Martínez, On interpolation of Banach algebras and factorization of weakly compact homomorphisms, Bull. Sci. Math. 130 (2006), 637-645.

[13] F. Cobos, L. M. Fernández-Cabrera and A. Martínez, Abstract K and J spaces and measure of non-compactness, Math. Nachr. 280 (2007), 1698-1708.

[14] J. B. Conway, A Course in Functional Analysis, Springer, New York, 1990.

[15] W. J. Davis, T. Figiel, W. B. Johnson and A. Pełczyński, Factoring weakly compact operators, J. Funct. Anal. 17 (1974), 311-327.

[16] J. E. Galé, T. J. Ransford and M. C. White, Weakly compact homomorphisms, Trans. Amer. Math. Soc. 331 (1992), 815-824.

[17] S. Heinrich, Closed operator ideals and interpolation, J. Funct. Anal. 35 (1980), 397-411.

[18] S. Janson, On interpolation of multilinear operators, in: Function Spaces and Applications, M. Cwikel, J. Peetre, Y. Sagher and H. Wallin (eds.), Lect. Notes in Math. 1302, Springer, Berlin 1988, 290-302. 
[19] S. Kaijser, Interpolation of Banach algebras and open sets, Integr. Equ. Oper. Theory 41 (2001), 189-222.

[20] M. Levy, L'espace d'interpolation réel $\left(A_{0}, A_{1}\right)_{\theta, p}$ contient $\ell^{p}$, Compt. Rend. Acad. Sci. Paris Sér. A 289 (1979), 675-677.

[21] L. Maligranda, Weakly compact operators and interpolation, Acta Appl. Math. 27 (1992), 79-89.

[22] L. Maligranda and A. Quevedo, Interpolation of weakly compact operators, Arch. Math. 55 (1990), 280-284.

[23] M. Mastyło, On interpolation of weakly compact operators, Hokkaido Math. J. 22 (1993), 105-114.

[24] P. Nilsson, Reiteration theorems for real interpolation and approximation spaces, Ann. Mat. Pura Appl. 132 (1982), 291-330.

[25] J. Peetre, A theory of interpolation of normed spaces, Lecture Notes, Brasilia, 1963 [Notes Mat. 39 (1968), 1-86].

[26] H. Triebel, Interpolation Theory, Function Spaces, Differential Operators, North-Holland, Amsterdam, 1978.

[27] M. Zafran, The dichotomy problem for homogeneous Banach algebras, Ann. Math. 108 (1978), 97-105. 
